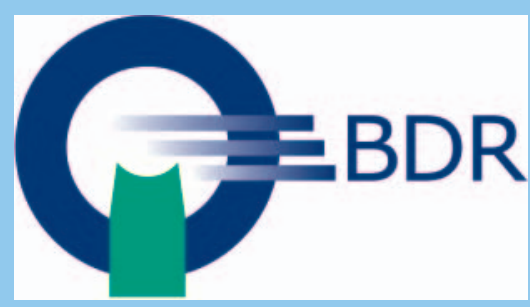

\title{
Berufspolitik 2021 - Einheit in Vielfalt
}

Liebe Kolleginnen und Kollegen,

im Wahljahr 2021 ist häufig vom veränderten Farbspektrum der Bundespolitik die Rede. Das berufspolitische Erscheinungsbild der Radiologen wurde schon im letzten Jahr deutlich bunter. Denn neben der DRG als Fachgesellschaft und dem BDR als Berufsverband haben sich seit Jahren weitere Gruppierungen etabliert, die sich speziellen Problemstellungen der Berufspolitik mit besonderem Interesse zuwenden. Und 2020 sind weitere dazugekommen.

So dient das Chefarztforum (CAFRAD) der DRG bereits seit Jahren als Netzwerk und Austauschforum für fachliche, organisatorische und juristische Anforderungen der Chefärzte in großen und kleinen Krankenhäusern. Denn trotz länderspezifischer Charakteristika sind in dieser Berufsgruppe viele Herausforderungen des Alltags bundesweit ähnlich. Das Forum Niedergelassener Radiologen (FuNRad) ist eine Plattform für den Ideen- und Erfahrungsaustausch in der radiologischen Niederlassung. Der Schwerpunkt liegt dabei auf Themen der klinischen und wissenschaftlichen Radiologie unter besonderer Berücksichtigung der Situation in der Praxis. Angesprochen sind sowohl selbständige als auch angestellte ärztliche Kolleginnen und Kollegen.

Die Erfahrungen bei der letzten Novellierung der Musterweiterbildungsordnung haben gezeigt, dass nur bei einer starken Vertretung unseres Faches in den gewähl- ten Gremien der Selbstverwaltung, wie Landesärztekammer, Landes-KV und von diesen nachfolgend zu besetzenden Gremien, Erfolge für die Radiologie zu erreichen sind. Hierzu bedarf es einer langfristigen Strategie um ausreichend Kolleginnen und Kollegen in den entsprechenden Delegiertenversammlungen zu verankern. Die neu gegründete AG Gesundheitspolitische Verantwortung der DRG ist dabei ein Forum zur Bündelung der unterschiedlichen Initiativen. Dass eine gezielte Planung dabei zum Erfolg führen kann, zeigte sich bei der letzten Kammerwahl in Baden-Württemberg.

Einem anderen Ziel hat sich die aus einer bayerischen Initiative hervorgegangene und inzwischen als eingetragene Genossenschaft bundesweit agierende RadiologenGruppe RG20 eG gesetzt. Sie möchte die speziellen Interessen von Großpraxen gegenüber KV und Politik vertreten und zusätzliche Wege zur Interessenwahrung im Vorfeld ausloten und ebnen.

Dabei ist die Strategie von Fachgesellschaft und Berufsverband sowie den genannten Gruppierungen ganz klar: nur gemeinsam können Erfolge für die Radiologie erzielt werden. Nur durch intensive Absprache untereinander kann die Stellung der Radiologie auf Dauer bestehen bleiben bzw. verbessert werden. So sind viele Akteure in mehr als einer der angesprochenen Gruppierungen aktiv. Besonders deutlich wurde dies in einer gemeinsamen Veranstaltung von BDR, CAFRAD und AG GPV im Rahmen der Reihe „Radiologie in Klinik und Praxis“ beim RöKo digital 2020 zur Kardiologischen Bildgebung. Hierbei wurden die unterschiedlichen Facetten des Themas von verschiedener Seite beleuchtet, geeint durch die Überzeugung, dass nur durch ein gemeinsames Vorgehen die Qualität der Untersuchungen hoch und die Durchführung damit innerhalb unseres Faches gehalten werden kann. Nichts wäre fataler als den anderen Fachrichtungen und den Gremien der Selbstverwaltung durch Zersplitterung leichtes Spiel zu bereiten.

„Alle Wege führen nach Rom.“ Dieser Ausspruch entstand anlässlich der Aufstellung einer goldenen Säule mit darauf verzeichneten Entfernungen von Rom in alle Provinzhauptstädte unter Kaiser Augustus. „Respektive nach Berlin“ möchte man heute umformulieren. Der BDR mit seinen Landesverbänden freut sich über jedes Engagement - in welcher Organisationsform auch immer - den oft steinigen Weg berufspolitischer Erfolge gemeinsam zu gehen.

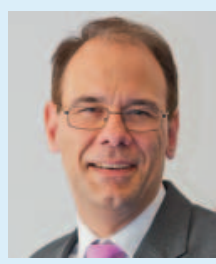

Prof. Hermann Helmberger München 